\title{
OBSTRUCTIONS TO THE EXISTENCE OF ALMOST COMPLEX STRUCTURES
}

\author{
BY W. S. MASSEY
}

Communicated June 9, 1961

1. Definitions and notation. Let $M$ be an orientable, differentiable manifold of dimension $2 n$ and let $\xi=\left(E_{\xi}, M, R^{2 n}, \pi\right)$ denote the tangent bundle of $M$; we assume the structural group of $\xi$ has been reduced from the full linear group to the special orthogonal group $S O(2 n)$. By definition, $M$ admits an almost complex structure if and only if the associated fibre bundle $\eta=\left(E, M, \Gamma_{n}, p\right)$ admits a cross section; ${ }^{1}$ here $\Gamma_{n}$ denotes the homogeneous space $S O(2 n) / U(n)$. In this paper, we will study the obstructions to a cross section for any fibre bundle $\theta=\left(E, B, \Gamma_{n}, p\right)$ with structural group $S O(2 n)$ and base space $B$ a $C W$-complex. If $s: B^{q} \rightarrow E$ is a cross section of $\theta$ over the $q$-skeleton of the base space $B$, then the obstruction to extending $s$ over the $(q+1)$-skeleton is denoted by

$$
c^{q+1}(s) \in H^{q+1}\left(B, \pi_{q}\left(\Gamma_{n}\right)\right) .
$$

Since $\theta$ is a bundle with structural group $S O(2 n)$, the following characteristic classes are defined:

(a) Integral Stiefel-Whitney classes,

$$
W_{i}(\theta) \in H^{i}(B, Z), \quad 3 \leqq i \leqq 2 n-1, \quad i \text { odd. }
$$

(Recall that $2 \cdot W_{i}(\theta)=0$.)

(b) Euler-Poincaré class, $W_{2 n}(\theta) \in H^{2 n}(B, Z)$.

(c) Pontrjagin classes $p_{i}(\theta) \in H^{4 i}(B, Z), 0 \leqq i \leqq n$.

In an analogous manner, if $\xi$ is a fibre bundle with base space $B$ and structural group $U(n)$, the Chern classes of $\xi$ will be denoted by $c_{i}(\xi) \in H^{2 i}(B, Z), 0 \leqq i \leqq n$.

2. Statement of results. The homotopy group $\pi_{q}\left(\Gamma_{n}\right)$ is called stable if $q<2 n-1$; it is well known that the stable homotopy groups $\pi_{q}\left(\Gamma_{n}\right)$ for fixed $q$ and variable $n$ are all isomorphic; see Gray [4, p. 432]. The stable homotopy groups of $\Gamma_{n}$ have been determined by Bott $[2]$; he showed that in the stable range,

${ }^{1}$ Standard references on the subject of almost complex structures are Ehresmann's lecture at the 1950 International Congress of Mathematicians [3] and the last section of Steenrod's book [10].

The author would like to take this opportunity to acknowledge that his proof of the two theorems announced in Abstract 60T-24, Notices Amer. Math. Soc. vol. 7 (1960) p. 1001, contains an apparently irreparable gap. Whether or not these two theorems are correct is not known. 


$$
\begin{aligned}
& \pi_{q}\left(\Gamma_{n}\right)=Z \\
& \pi_{q}\left(\Gamma_{n}\right)=Z_{2} \\
& \pi_{q}\left(\Gamma_{n}\right)=0
\end{aligned}
$$$$
\text { for } q \equiv 2 \bmod 4 \text {, }
$$$$
\text { for } q \equiv 0 \text { or }-1 \bmod 8 \text {, }
$$$$
\text { for all other values of } q \text {. }
$$

LEMMA 1. The first nonstable homotopy group $\pi_{2 n-1}\left(\Gamma_{n}\right)(n>0)$ is as follows:

$$
\pi_{2 n-1}\left(\Gamma_{n}\right)= \begin{cases}\boldsymbol{Z}+\boldsymbol{Z}_{2} & \text { for } n \equiv 0 \bmod 4 \\ \boldsymbol{Z}_{(n-1) !} & \text { for } n \equiv 1 \bmod 4, \\ \boldsymbol{Z} & \text { for } n \equiv 2 \bmod 4, \\ \boldsymbol{Z}_{(n-1) ! / 2} & \text { for } n \equiv 3 \bmod 4 .\end{cases}
$$

This result for $n \equiv 0 \bmod 4$ is due to Bruno Harris. The results for the other three cases are easier; they are proved by considering the homotopy sequences of some well-known fibre bundles and using the results listed in a paper of Kervaire [9].

Recall that the vanishing of the integral Stiefel-Whitney classes $W_{2 q+1}(\theta)$ is a well-known necessary condition for the existence of a cross section of the fibre bundle $\theta=\left(E, B, \Gamma_{n}, p\right)$ (see Steenrod [10, p. 212]). On the other hand in the stable range the obstruction to a cross section in dimension $4 k+3$ will be an integral cohomology class in view of (1). It is natural to conjecture that there should be some relation between this obstruction and the Stiefel-Whitney class $W_{4 k+3}(\theta)$.

TheOREM I. Let $s: B^{q} \rightarrow E$ be a cross section of the bundle $\theta$ over the $q$-skeleton, where $q=4 k+2$ and $q<2 n-1$. Then

$$
W_{q+1}(\theta)=\left\{\begin{array}{cc}
(2 k) ! c^{q+1}(s) & \text { for } k \text { even, } \\
(1 / 2)(2 k) ! c^{q+1}(s) & \text { for } k \text { odd. }
\end{array}\right.
$$

REMARK 1. For $q=2$, this theorem asserts that $W_{3}(\theta)=c^{3}(s)$, which is of course well known. For $q=6$, the result becomes $W_{7}(\theta)=c^{7}(s)$, a result announced by Ehresmann without proof in 1950 [3].

REMARK 2. In case $H^{q+1}(B, \boldsymbol{Z})$ has no $p$-torsion for any prime $p \leqq 2 k$, then the condition $W_{q+1}(\theta)=0$ implies that $c^{q+1}(s)=0$.

REMARK 3. This theorem bears a slight similarity to formula (ii) of Lemma $(1,1)$ of Kervaire [8]. The proof here is more difficult because $\Gamma_{n}$ is not a topological group and $\theta$ is not a principal bundle.

REMARK 4. This theorem implies divisibility conditions on the integral Stiefel-Whitney classes. For example, if $\theta=(E, B, p)$ is a bundle with group $S O(2 n), n \geqq 6$, such that $H^{8}\left(B, Z_{2}\right)=H^{9}\left(B, Z_{2}\right)=0$ and $W_{3}(\theta)=W_{7}(\theta)=0$, then $W_{11}(\theta)$ is divisible by 24 . 
As motivation for the next theorem, recall that if the bundle $\theta$ admits a cross section $s$, then the structural group can be reduced from $S O(2 n)$ to the subgroup $U(n)$. Let $\xi$ denote the $U(n)$ bundle thus defined ( $\xi$ depends on the cross section $s$ ) and $c_{i}(\xi), 1 \leqq i \leqq n$, its Chern classes. Then the following relations must hold between the Pontrjagin classes $p_{i}(\theta)$ and the Chern classes $c_{i}(\xi)$ :

$$
(-1)^{k} p_{k}(\theta)=\sum_{i+j=2 k}(-1)^{i} c_{i}(\xi) c_{j}(\xi), \quad 0 \leqq k \leqq n
$$

(see Hirzebruch, $[6$, Satz 4.5.1, p. 68]). In addition, the top Chern class and the Euler-Poincaré class are equal:

$$
W_{2 n}(\theta)=c_{n}(\xi) .
$$

Now assume that $n$ is even, $n=2 k$, and that $s: B^{2 n-1} \rightarrow E$ is a cross section of $\theta$ over the $2 n-1$ skeleton. The obstruction $c^{2 n}(s)$ $\in H^{2 n}\left(B, \pi_{2 n-1}\left(\Gamma_{n}\right)\right)$ is an integral class if $n \equiv 2 \bmod 4$, while $c^{2 n}(s)$ $=c_{0}^{2 n}(s)+c_{2}^{2 n}(s)$ if $n \equiv 0 \bmod 4$, where $c_{0}^{2 n}$ is an integral class and $c_{2}^{2 n}$ is a mod 2 cohomology class.

THEOREM II. For $n=2 k, k$ odd,

$$
\sum_{i+j=2 k}(-1)^{i} c_{i}(\xi) c_{j}(\xi)-(-1)^{k} p_{k}(\theta)=4 \cdot c^{2 n}(s)
$$

while for $n=2 k, k$ even, this same formula holds true with $c^{2 n}(s)$ replaced by its integral component, $c_{0}^{2 n}(s)$. In this formula, $c_{0}(\xi), \cdots, c_{n-1}(\xi)$ are the Chern classes of the $U(n)$ bundle $\xi$ induced over $B^{2 n-1}$ by s, while $c_{n}(\xi)=W_{2 n}(\theta)$.

Theorems I and II give information about the obstructions to a cross section of $\theta$ in all cases of importance where the coefficient group is infinite cyclic. Further information is needed in case the coefficient group is $Z_{2}$. The first such case is the following: Assume $s: B^{7} \rightarrow E$ is a cross section of $\theta=\left(E, B, \Gamma_{n}, p\right)$ over the 7 -skeleton and $n>4$. Then $c^{8}(s)$ is a mod 2 cohomology class. The existence of $s \mathrm{im}-$ plies that $W_{3}(\theta), W_{5}(\theta)$, and $W_{7}(\theta)$ vanish, and that the fibre $\Gamma_{n}$ is totally nonhomologous to zero in dimensions $\leqq 8$ with any coefficients. ${ }^{2}\left(H^{*}\left(\Gamma_{n}, \boldsymbol{Z}\right)\right.$ is torsion free.) In dimensions $\leqq 8, H^{*}\left(\Gamma_{n}, \boldsymbol{Z}\right)$ is a polynomial ring ${ }^{2}$ on generators $x \in H^{2}\left(\Gamma_{n}, Z\right)$ and $y \in H^{6}\left(\Gamma_{n}, Z\right)$. It follows that there exist elements $u \in H^{2}(E, Z)$ and $v \in H^{6}(E, Z)$ such that

$$
i^{*}(u)=x, \quad i^{*}(v)=y,
$$

where $i: \Gamma_{n} \rightarrow E$ is the inclusion map.

2 These assertions follow easily from the facts about the cohomology of $\Gamma_{n}$ stated in the next section. 
Lemma 2. Given the cross section $s$, it is possible to choose $u$ and $v$ so that (4) is satisfied and $s^{*}(u)=s^{*}(v)=0$. Conversely, given the cohomology classes $u$ and $v$ satisfying (4), there exists a cross section $s: B^{7} \rightarrow E$ such that $s^{*}(u)=s^{*}(v)=0$.

Next, it may be shown that $S q^{2} y=x^{4}(\bmod 2)$. Since the fibre is totally nonhomologous to 0 in dimensions $\leqq 8$, there exist unique mod 2 cohomology classes $b_{2}, b_{2}^{\prime}, b_{4}, b_{6}, b_{8}$ on $B$ such that

$$
\begin{aligned}
S q^{2} v= & u^{4}+p^{*}\left(b_{8}\right)+p^{*}\left(b_{6}\right) \cdot u+p^{*}\left(b_{4}\right) \cdot u^{2} \\
& +p^{*}\left(b_{2}\right) \cdot u^{3}+p^{*}\left(b_{2}^{\prime}\right) \cdot v(\bmod 2)
\end{aligned}
$$

(the subscripts denote the degree).

THEOREM III. If $u$ and $v$ are chosen to satisfy (4) and $s^{*}(u)=s^{*}(v)$ $=0$, and $b_{8} \in H^{8}\left(B, Z_{2}\right)$ is chosen to satisfy (5), then

$$
c^{8}(s)=b_{8} .
$$

This theorem essentially asserts that determination of $c^{8}(s)$ requires the computation of $S q^{2}: H^{6}(E, \boldsymbol{Z}) \rightarrow H^{8}\left(E, \boldsymbol{Z}_{2}\right)$, where $H^{*}(E)$ is considered as a module over $H^{*}(B)$. This computation is at present a very difficult problem.

An easy computation using Lemma 2 shows that if $s_{0}, s_{1}: B^{7} \rightarrow E$ are cross sections, then there exist cohomology classes $d_{2} \in H^{2}(B, Z)$ and $d_{6} \in H^{6}(B, \boldsymbol{Z})$ such that

$$
c^{8}\left(s_{0}\right)-c^{8}\left(s_{1}\right)=S q^{2} d_{6}+\left(d_{2}\right)^{4}(\bmod 2) .
$$

Moreover, given $s_{0}, d_{2}$, and $d_{6}$, there exists a cross section $s_{1}$ such that this equation holds true.

Corollary. If $\theta=\left(E, B, \Gamma_{n}, p\right)$ is a bundle with structural group $S O(2 n), n>4$ such that $W_{3}(\theta)=W_{7}(\theta)=0$ and

$$
H^{8}\left(B, Z_{2}\right)=S q^{2} H^{6}(B, Z)+S q^{4} S q^{2} H^{2}(B, Z)
$$

then $\theta$ admits a cross section over the 8-skeleton of $B$.

3. Some remarks on the proof of these theorems. We use the method of $\mathrm{R}$. Hermann [5] to study the obstructions to cross sections of the bundle $\theta=\left(E, B, \Gamma_{n}, p\right)$. This method utilizes a Moore-Postnikov decomposition of the fibre space $\theta$, which in turn requires some knowledge of a Postnikov decomposition of the fibre $\Gamma_{n}$. In order to use a Postnikov decomposition of $\Gamma_{n}$ it is necessary to study the cohomology of $\Gamma_{n}$. The following are the relevant facts:

(a) $\Gamma_{n}$ is torsion free; additively, its integral cohomology groups 
are isomorphic to those of the following product of even dimensional spheres:

$$
S^{2} \times S^{4} \times S^{6} \times \cdots \times S^{2 n-2}
$$

(see Borel, [1, p. 203]).

(b) The integral cohomology ring $H^{*}\left(\Gamma_{n}, Z\right)$ has a simple system of generators (in the sense of Borel, [1, p. 141]) $\alpha_{1}, \alpha_{2}, \cdots, \alpha_{n-1}$, with $\alpha_{i}$ of degree $2 i$. These generators satisfy the following relations, which completely determine the structure of the integral cohomology ring:

$$
\begin{aligned}
& \alpha_{1}^{2}-\alpha_{2}=0, \\
& \alpha_{2}^{2}-2 \alpha_{1} \alpha_{3}+\alpha_{4}=0, \\
& \alpha_{3}^{2}-2 \alpha_{2} \alpha_{4}+2 \alpha_{1} \alpha_{5}-\alpha_{6}=0, \\
& \vdots \\
& \dot{2}_{n-2}^{2}-2 \alpha_{n-3} \alpha_{n-1}=0, \\
& \alpha_{n-1}^{2}=0 .
\end{aligned}
$$

(c) In any fibre bundle $\theta=\left(E, B, \Gamma_{n}, p\right)$ with group $S O(2 n)$, the generators $\alpha_{1}, \cdots, \alpha_{n-1}$ listed above are transgressive; the transgression of the generator $\alpha_{i}$ is the integral Stiefel-Whitney class $W_{2 i+1}(\theta)$ (modulo the ideal generated by $W_{3}, \cdots, W_{2 i-1}$ ).

(d) In the fibre space $p: B_{U(n)} \rightarrow B_{S O(2 n)}$ determined by the inclusion $U(n) \subset S O(2 n)$, the homomorphism $i^{*}: H^{*}\left(B_{U(n)}, Z\right) \rightarrow H^{*}\left(\Gamma_{n}, Z\right)$, where $i: \Gamma_{n} \rightarrow B_{U(n)}$ is the inclusion map, satisfies

$$
\begin{array}{ll}
i^{*}\left(c_{j}\right)=2 \alpha_{j}, & 1 \leqq j \leqq n-1,
\end{array}
$$

Here $c_{j} \in H^{2 j}\left(B_{U(n)}, Z\right)$ denotes the universal Chern class.

(e) From (c), one can determine the Steenrod squares in $H^{*}\left(\Gamma_{n}, \boldsymbol{Z}_{2}\right)$. It is necessary to reduce modulo 2 and use the known formulas of $\mathrm{W}$. T. Wu for the squares of the Stiefel-Whitney classes.

(f) It follows from (d) that $i^{*}: H^{*}\left(B_{U(n)}, Z_{p}\right) \rightarrow H^{*}\left(\Gamma_{n}, Z_{p}\right)$ is a homomorphism onto for any odd prime $p$; hence the formulas of Serre and Borel for the Steenrod reduced powers in $H^{*}\left(B_{U(n)}, \boldsymbol{Z}_{p}\right)$ determine those in $H^{*}\left(\Gamma_{n}, \boldsymbol{Z}_{p}\right)$.

From these facts, one can determine enough information about the Postnikov invariants of $\Gamma_{n}$ to prove Theorems I, II and III by Hermann's method; full details will be published elsewhere. ${ }^{3}$

${ }^{3}$ These results may perhaps be regarded as a first small step in the program mentioned by Hirzebruch in the middle of p. 127 of his 1958 lecture [7]. 
Note that all our results.extend to analogous theorems about the almost contact manifolds of Gray [4]; for example, an orientable 7 -dimensional manifold admits an almost contact structure if and only if $W_{3}=0$ (since $W_{7}=0$ automatically).

\section{BIBLIOGRAPHY}

1. A. Borel, Sur la cohomologie des espaces fibres principaux et des espaces homogènes de groupes de Lie compacts, Ann. of Math. vol. 57 (1953) pp. 115-207.

2. R. Bott, The stable homotopy of the classical groups, Ann. of Math. vol. 70 (1959) pp. 313-338.

3. C. Ehresmann, Sur les variêtés presque complexes, Proceedings of the International Congress of Mathematicians, vol. II (1950) pp. 412-419.

4. J. W. Gray, Some global properties of contact structures, Ann. of Math. vol. 69 (1959) pp. 421-450.

5. R. Hermann, Secondary obstructions for fibre spaces, Bull. Amer. Math. Soc. vol. 65 (1959) pp. 5-8.

6. F. Hirzebruch, Neue topologische Methoden in der algebraischen Geometrie, Ergebnisse der Mathematik, vol. 9 (1956)

7. - Komplexe Mannigfaltigkeiten, Proceedings of the International Congress of Mathematicians (1958) pp. 119-136.

8. M. Kervaire, $A$ note on obstructions and characteristic classes, Amer. J. Math. vol. 81 (1959) pp. 773-784.

9. - Some non-stable homotopy groups of Lie groups, Illinois J. Math. vol. 4 (1960) pp. 161-170.

10. N. E. Steenrod, The topology of fibre bundles, Princeton University Press, 1951.

YALE UNIVERSITY 